\title{
SOP Untersuchung von pädiatrischen Notfallpatienten
}

Thomas Gaisch, Martin Fandler, Christian Joachim

\begin{abstract}
Kindernotfälle sind selten, gerade deshalb lohnt es aber, sich im Vorhinein mit dem Thema auseinanderzusetzen, um strukturierte Untersuchungs- und Behandlungskonzepte auch in stressigen Situationen parat zu haben und umsetzen zu können. Denn: Kinder sind keine kleinen Erwachsenen. Neben Unterschieden in Anatomie und Physiologie brauchen Kinder gerade in Notfallsituationen viel Feingefühl bei Untersuchung und Therapie.
\end{abstract}

\section{Strukturiertes Vorgehen}

Das ABCDE-Schema ist bereits von der Untersuchung Erwachsener vertraut und kann auch für Kinder effektiv angewandt werden ( $\bullet$ Abb. 1). Wichtig ist ein strukturiertes Vorgehen von A nach $E$, damit keine Details übersehen werden und nicht unnötig Zeit durch chaotische Abläufe verloren wird. Für Kindernotfälle besonders relevante $\mathrm{Er}$ krankungen werden in der vorliegenden SOP nach den Kategorien „häufig“ sowie „selten, aber gefährlich“ aufgelistet und sollten im Einsatz strukturiert abgearbeitet werden.

Das für die Einschätzung der Vitalwerte ( $\triangleright$ Tab. 1$)$ wichtige Körpergewicht der Kinder sollte von den Eltern erfragt werden, es stehen auch verschiedene Hilfsmittel (z. B. Notfall-Lineal) zur Verfügung.

Merke

Vorab überlegte Untersuchungsabläufe bieten

Sicherheit und sparen Zeit!

\section{Klinische Untersuchung}

\section{Airway und Breathing}

Bei Kindern führen Probleme im Bereich des Atemweges häufig zu Notfällen und beeinflussen dadurch die anderen Vitalfunktionen erheblich.

Neben Standardmonitoring mit $\mathrm{SpO}_{2}$, EKG und Blutdruckmessung liefern auch klinische Untersuchungen wie Inspektion und Auskultation nützliche Informationen. Dyspnoe, Tachypnoe, Orthopnoe und Zyanose lassen auf ein respiratorisches Problem schließen. Inspiratorischer Stridor deutet auf ein Problem der oberen Atemwege wie etwa bei Pseudokrupp - hin, während exspiratorischer Stridor eher die unteren Atemwege - wie z. B. im
> Tab. 1 Kinder-Vitalwerte.

\begin{tabular}{|c|l|l|l|}
\hline Gewicht & Alter (ca.) & Herzfrequenz & Atemfrequenz \\
\hline $3 \mathrm{~kg}$ & Neugeborene & 140 & 40 \\
\hline $5 \mathrm{~kg}$ & < 1 Jahr & 130 & 35 \\
\hline $10 \mathrm{~kg}$ & 1-2 Jahre & 120 & 30 \\
\hline $15 \mathrm{~kg}$ & 3-4 Jahre & 110 & 25 \\
\hline $20 \mathrm{~kg}$ & 5-6 Jahre & 100 & 20 \\
\hline $25 \mathrm{~kg}$ & 7-8 Jahre & 95 & 20 \\
\hline $30 \mathrm{~kg}$ & 9-10 Jahre & 90 & 20 \\
\hline
\end{tabular}

Falle einer Bronchiolitis - betrifft. Bei viralen Erkrankungen wie Pseudokrupp entsteht häufig bellender Husten, zusätzliche thorakale Einziehungen deuten auf erhöhten Atemaufwand hin und sind je nach Ausprägung von unterschiedlichem Schweregrad. Zuerst entstehen juguläre, im Verlauf dann auch subkostale Einziehungen. In der schwersten Ausprägung zeigen sich schließlich subxiphoidale Einziehungen, was bereits auf massive Atemnot schließen lässt.

\section{Circulation}

Bradykardie und im schlimmsten Fall auch Asystolie treten häufig als Folge von Hypoxie auf. Tachykardie kann durch Exsikkose im Rahmen von Durchfallerkrankungen oder hohem Fieber auftreten. Stehende Hautfalten sind ein spätes Zeichen, hilfreich können neben Anamnese auch trockene Schleimhäute oder eine eingesunkene Fontanelle sein. Bei unklarer Tachykardie und Hypotension sollte auch an eine mögliche Anaphylaxie gedacht werden, auch hohe Blutverluste rufen Tachykardien hervor. Kinder können eine Hypovolämie verhältnismäßig lange kompensieren und wirken klinisch noch unauffällig - dekompensieren dann allerdings schnell und fulminant. 


\section{Diagnostik}
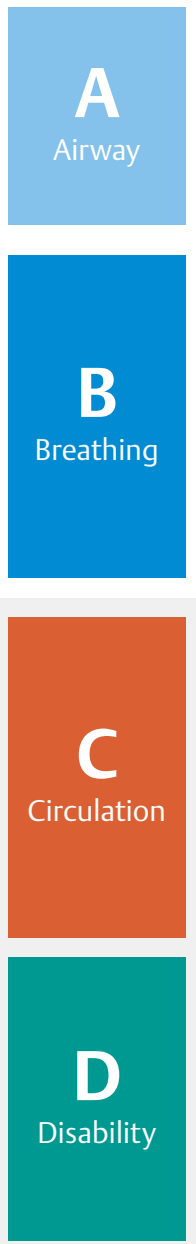

Bewusstsein eingeschränkt (adaptierte GCS für Kinder) Meningismus prüfen Pupillen Zungenbiss/Sezessus Blutzucker messen

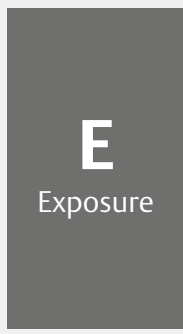

Fieber messen!

(Bauch-)Schmerzen?

Exanthem/Petechien

Verletzungen

U-Heft/Impfpass/Mutter-Kind-Pass Umgebung/Eltern

- Abb. 1 ABCDE-Schema.

Schnell und einfach sollte daher auch die Rekapillarisierungszeit getestet werden, die oft ein frühes Warnzeichen eines Schocks darstellt. Diese sollte unter 2 Sekunden liegen.

Cave

Selten, aber wichtig: Schlechtes Trinkverhalten und Zyanose in den ersten Lebenstagen weisen auf angeborene Herzfehler hin!
Erkrankungen
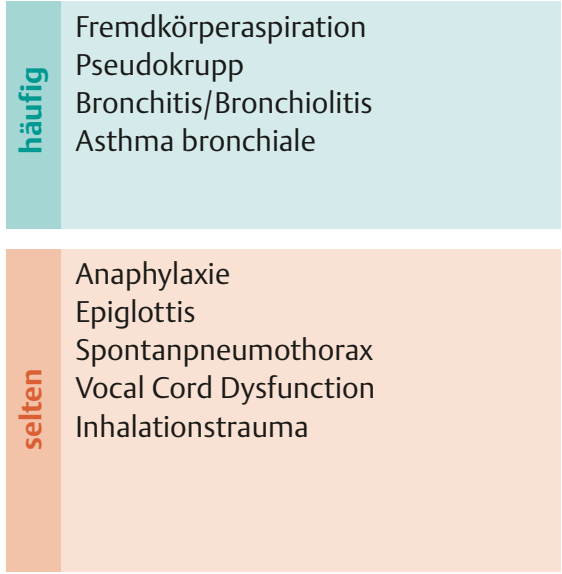

믄 Bradykardie durch Hypoxie

Exsikkose

도
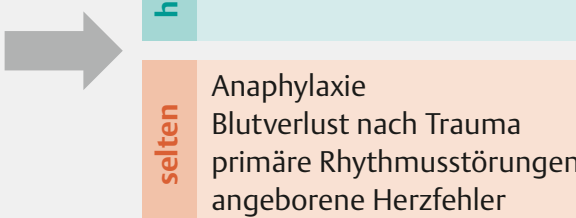

angeborene Herzfehler

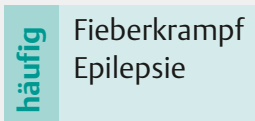

ᄃ Diabetes mellitus (Erstmanifestation)

Meningitis (Meningokokken!)

Hirnblutung

\section{Infektionen v.a. viral \\ 믄 Appendizitis/akutes Abdomen}

: Verletzungen nach Trauma

Intoxikationen

Anaphylaxie

IJ Kindesmisshandlung

Sepsis

\section{Disability}

Die Glasgow Coma Scale sollte adaptiert für Kinder verwendet werden (siehe $>$ Tab. 2). Kindliche Bewusstseinstrübungen treten häufig bei Fieberkrämpfen auf, sind aber zumeist nur passager. Differenzialdiagnostisch weisen postiktale Phase, Zungenbiss oder Harn- bzw. Stuhlabgang auf eine potenzielle Epilepsie hin. 
Auch Erstmanifestationen eines Diabetes mellitus führen zu Störungen des Bewusstseins, weshalb die rasche Blutzuckerbestimmung - wie auch bei Erwachsenen- ausnahmslos bei der Diagnostik von unklarer Bewusstlosigkeit durchgeführt werden muss.

Meningitis bei Kindern ist verhältnismäßig selten - bestehen allerdings neben Fieber und etwaigem Meningismus auch Bewusstseinstrübung und Petechien, sollten zeitkritisch Antibiotika verabreicht werden.

\section{Nichts übersehen}

\section{Exposure}

Infektionen, Intoxikationen, Verletzungen, Exantheme oder Petechien können durch gründliche Untersuchung und gute Anamnese entdeckt werden.

Wenn es die Situation erfordert, sollten Kinder von oben bis unten untersucht werden. Allerdings muss hierbei der Wärmeerhalt gerade bei Neugeborenen und Säuglingen stets im Auge behalten werden. Die Messung der Temperatur sollte spätestens an diesem Untersuchungsschritt durchgeführt werden.

Die Abschätzung von Schmerzen ist bei kleinen Kindern oft schwierig.

Bei ungewöhnlichen Verletzungen muss auch stets an Kindesmisshandlung gedacht werden! (Passen Verletzungsmuster und Anamnese zusammen?)

\section{Merke}

An Wärmemanagement denken!

\section{Umgebung}

Auch wenn das professionelle Personal nur selten mit Kindernotfällen zu tun hat - die betroffenen Kinder und Eltern noch deutlich seltener! Eine der zentralen Rollen des Rettungsdienstes bei Kindernotfällen ist daher die Ausstrahlung von Ruhe und Sicherheit.

Eltern können und sollen - sofern sie Untersuchung und Therapie nicht behindern - auch in Notfallsituationen eingebunden werden. Gerade kleinere Kinder profitieren von der beruhigenden Wirkung durch die Anwesenheit der Eltern. Zudem können Eltern bei der Anamnese weiterhelfen und wissen oft das Gewicht ihres Kindes, was für Medikamentenberechnungen nützlich sein kann. Auch Geschwister bieten oft nützliche Informationen sind diese ebenfalls erkrankt, wurden Spielsachen verschluckt, haben sie mit Tabletten gespielt etc.? Einen Blick wert sind übrigens auch U-Heft, ggf. auch Impfpass sowie Mutter-Kind-Pass.
Tab. 2 Erweiterter Pädiatrischer Glasgow Coma Scale (Original siehe [5]).

\begin{tabular}{|c|c|c|}
\hline & Reaktion & Punkte \\
\hline \multirow[t]{4}{*}{ Augen öffnen } & spontan & 4 \\
\hline & auf Ansprache & 3 \\
\hline & auf Schmerzreiz & 2 \\
\hline & keine & 1 \\
\hline \multirow{5}{*}{$\begin{array}{l}\text { verbale } \\
\text { Kommunikation }\end{array}$} & Plappern, Brabbeln & 5 \\
\hline & Schreien, tröstbar & 4 \\
\hline & Schreien, untröstbar & 3 \\
\hline & Stöhnen/unverständliche Laute & 2 \\
\hline & keine & 1 \\
\hline \multirow{6}{*}{$\begin{array}{l}\text { motorische } \\
\text { Reaktion }\end{array}$} & spontan & 6 \\
\hline & auf Schmerzreiz gezielt & 5 \\
\hline & auf Schmerzreiz ungezielt & 4 \\
\hline & auf Schmerzreiz abnormale Beugung & 3 \\
\hline & auf Schmerzreiz Strecksynergismen & 2 \\
\hline & keine & 1 \\
\hline
\end{tabular}

FAZIT

- Ruhe bewahren

- Eltern sofern möglich einbinden

- Kindernotfälle sind selten, deshalb sollten bereits im Vorfeld Konzepte bestehen

- Kinder nur wenn nötig ausführlich, dann aber „von oben bis unten“ untersuchen

Interessenkonflikt

Die Autoren geben an, dass kein Interessenkonflikt besteht.

\section{Autorinnen / Autoren}

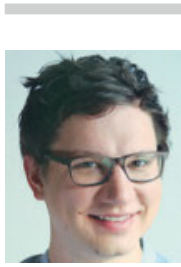

\section{Thomas Gaisch}

Dr. med. univ. 2006-2013 Studium der Humanmedizin an der Medizinischen Universität Graz. 2014-2015 Universitätsklinik für Notfallmedizin und internistische Intensivmedizin Nürnberg, seit 2015 Universitätsklinik für Anästhesiologie und operative Intensivmedizin Nürnberg, Paracelsus Medizinische Privatuniversität.

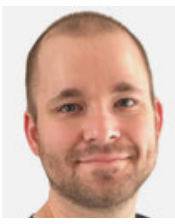

\section{Martin Fandler}

Dr. med. univ. 2006-2012 Studium der Humanmedizin an der Medizinischen Universität Graz; seit 2012 am Klinikum Nürnberg tätig. Auslandsaufenthalte in Notfallzentren in den USA und Südafrika. Arbeitsschwerpunkte in der zentralen Notaufnahme und Notarztdienst. 


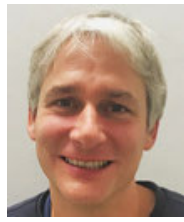

\section{Christian Joachim}

Dr. med. univ. Studium Humanmedizin Semmelweis Universität Budapest. Abschluss 1998. Facharzt für Kinder- und Jugendheilkunde und Facharzt für Anästhesiologie. Pädiatrische Intensivmedizin in London von 2006-2012, u. a. Royal London Hospital. Seit 2012 Paracelsus Medizinische Privatuniversität Nürnberg, Universitätsklinik für Anästhesiologie und operative Intensivmedizin.

Korrespondenzadresse

Dr. med. univ. Thomas Gaisch

Universitätsklinik für Anästhesiologie

und operative Intensivmedizin

Paracelsus Medizinische Privatuniversität Nürnberg

Breslauer Str. 201

90471 Nürnberg

thomas.gaisch@klinikum-nuernberg.de

\section{Literatur}

[1] Nicolai T. Pädiatrische Notfall- und Intensivmedizin. 5. Aufl. Berlin, Heidelberg: Springer; 2014

[2] Müller R et al. Medikamente und Richtwerte in der Notfallmedizin. 11. Aufl. Graz: Ralf Müller Verlag; 2012

[3] Flake F, Scheinichen F. Kindernotfälle im Rettungsdienst. 5. Aufl. Berlin, Heidelberg: Springer; 2016

[4] Fleming S, Thompson M, Stevens R et al. Normal ranges of heart rate and respiratory rate in children from birth to 18 years of age: a systematic review of observational studies. Lancet 2011; 377: 1011

[5] Holmes JF, Palchak M], MacFarlane T et al. Performance of the pediatric Glasgow coma scale in children with blunt head trauma. Acad Emerg Med 2005; 12: 814

[6] Praktische Guidelines und SOPs für Kindernotfälle (Englisch). Im Internet: http://site.cats.nhs.uk/in-a-hurry/cats-clinicalguidelines; Stand: 10.11.2017

[7] ALS für Neugeborene und Kinder, ERC Guidelines Stand 2015. Im Internet: http://www.grc-org.de/leitlinien2015; Stand: 10.11.2017

Bibliografie

DOI https://doi.org/10.1055/s-0043-122294

Notfallmedizin up2date 2017; 12: 347-350

(c) Georg Thieme Verlag KG Stuttgart · New York ISSN 1611-6550 